\title{
Déterminants de la qualité pour la mangue produite dans le Nordeste brésilien
}

\author{
Cécile Arnaud $^{a *}$, Henri Vannière ${ }^{b}$, Thierry Goguey ${ }^{b}$
}

\author{
a Centre national d'études \\ agronomiques des régions \\ chaudes (Cnearc), \\ av. agropolis, \\ 34000 Montpellier, \\ France \\ cecilearnaud@hotmail.com \\ ${ }^{\mathrm{b}}$ Cirad-flhor, TA 50 / PS4, \\ bld. De la Lironde, \\ 34398 Montpellier Cedex 5, \\ France \\ henri.vanniere@cirad.fr \\ thierry.goguey_muethon@ \\ cirad.fr
}

* Correspondance et tirés à part

Reçu le 28 avril 2002

Accepté le 10 février 2003

Fruits, 2003, vol. 58, p. 191-204 (C) 2003 Cirad/EDP Sciences All rights reserved

DOI: $10.1051 /$ fruits:2003007

RESUMEN ESPAÑOL, p. 204

\section{Determinants of quality for mango produced in the Brazilian Nordeste.}

Abstract - Introduction. Since the installation, in the sixties, of vast irrigated perimeters in the Petrolina/Juazeiro area (Nordeste, Brazil), the region has become one of the large Brazilian areas of fruit-bearing production and, in particular, of mangos intended for export. In this zone, the producer-privileged situation is, however, threatened by prospects for very strong competition on the internal and export markets. The purchasers could become more demanding regarding the fruit quality. The study presented was undertaken to highlight the production organization and mango distribution to understand how quality could influence the operators' strategies in this zone. Materials and methods. An investigation carried out for 4 months in the Petrolina/Juazeiro region and into the largest wholesale Brazilian market located in São Paulo made it possible to consult 50 professional operators: producers, people in charge of packaging, wholesalers, exporters, importers, scientists and public institution representatives. Results and discussion. The distribution flows of mango produced in the Petrolina/Juazeiro region indicate that a strong production proportion (65\%) is sold on the internal market and that $55 \%$ is forwarded by the wholesale markets. The successive exchange circuits, prejudicial to the fruit quality, can be very long. Analysis of the marketing and production costs shows that, at certain periods of the year, the internal market can be as remunerative as the market of exports towards Europe. The American market is that which involves most risks because of the significant investments it imposes, but it is also potentially the most lucrative from August to September. The quality of Petrolina/Juazeiro mango is due to its appearance, and especially to the management of maturity. Conclusion. Today prices are more determined by quantities available on the markets than by the effective mango quality. Because of competition to come, this situation should not last and quality will be essential to remaining competitive on the market. Better coordination of the mango operators and the development of techniques or training for better determination of the ideal time of harvesting and better control of the development of the fruit after harvest would make it possible to solve the most serious problems of quality.

Brazil / Mangifera indica (fruits) / production / marketing channels / quality / market information

\section{Déterminants de la qualité pour la mangue produite dans le Nordeste brésilien.}

Résumé - Introduction. Depuis la mise en place, dans les années 60, de vastes périmètres irrigués dans la région de Petrolina/Juazeiro (Nordeste, Brésil), celle-ci est devenue l'un des grands pôles brésiliens de production fruitière et, en particulier, de mangues destinées à l'exportation. La situation privilégiée des producteurs de cette zone est pourtant menacée par des perspectives de concurrence très forte sur les marchés internes et d'exportation. Les acheteurs pourraient devenir plus exigeants vis-à-vis de la qualité des fruits. L'étude présentée a cherché à mettre en évidence l'organisation de la production et de la distribution des mangues pour comprendre comment la qualité pouvait influencer les stratégies des opérateurs de cette zone. Matériel et méthodes. Une enquête réalisée pendant 4 mois sur le site de Petrolina/Juazeiro et sur le plus grand marché de gros brésilien à São Paulo a permis d'interroger 50 opérateurs de la filière: producteurs, responsables de stations d'emballages, grossistes, exportateurs, importateurs, scientifiques et représentants d'institutions publiques. Résultats et discussion. La répartition des flux de mangues produites à Petrolina/Juazeiro indique qu'en général une forte proportion $(65 \%)$ de la production est vendue sur le marché interne et que $55 \%$ transitent par les marchés de gros. Les circuits d'échanges successifs, préjudiciable à la qualité des fruits, peuvent être très longs. La décomposition des coûts de production et de commercialisation montre que, à certaines périodes de l'année, le marché interne peut être aussi rémunérateur que le marché d'exportation vers l'Europe. Le marché américain est celui qui comporte le plus de risques en raison des investissements importants à réaliser, mais il est aussi potentiellement le plus lucratif d'août à septembre. La qualite des mangues produites à Petrolina/Juazeiro tient à leur aspect et surtout à la gestion de la maturité. Conclusion. Aujourd'hui les prix sont davantage déterminés par les quantités disponibles sur les marchés que par la qualité effective des mangues. En raison de la concurrence à venir, cette situation ne devrait pas durer et une meilleure gestion de la qualité sera bientôt essentielle pour rester compétitif sur le marché. Une meilleure coordination des acteurs de la filière et le développement de techniques ou de formations pour mieux déterminer le point de cueillette idéal et mieux contrôler le développement des fruits après la coupe permettraient de résoudre les problèmes de qualité les plus sérieux.

Brésil / Mangifera indica (fruits) / production / circuit de commercialisation / qualité / information sur le marché 


\section{Introduction}

Au Brésil, près de $30 \%$ de la production fruitière et la moitié des exportations de fruits frais proviennent de la région du Nordeste. Dans cette zone, les cultures fruitières ayant la plus forte valeur commerciale sont l'oranger, le manguier, le raisin et le melon.

Au Brésil, la production de mangues s'est élevée à environ 700000 t en 2000. Elle provient principalement de la région de São Paulo et des états du Nordeste comme Bahia, le Minas Gerais, et le Pernambuco. $\mathrm{Au}$ cours de ces dernières années, les exportations de mangues ont rapidement augmenté. Les volumes exportés, insignifiants en 1980, ont atteint près de 40000 t en 1998, plaçant la mangue au premier rang des fruits frais exportés par le Brésil. L'évolution s'est poursuivie ensuite, la production dépassant les $67000 \mathrm{t}$ en 2000 , ce qui correspond à un chiffre d'affaire de 35,7 M US\$ [1, 2].

\subsection{Contexte de la production}

Jusqu'à une époque récente, les conditions climatiques du Nordeste brésilien caractérisées par de faibles précipitations [(400 à 800) $\mathrm{mm} \cdot \mathrm{an}^{-1}$ ] concentrées sur 4 mois et suivies de sécheresses régulières entravaient le développement agricole. C'est pourquoi, les gouvernements brésiliens qui se sont succédés depuis la fin du XIX ${ }^{e}$ siècle ont tenté d'apporter des solutions aux difficultés rencontrées dans cette région et, en particulier, dans la zone du Sertão semi-aride. Ainsi, à partir de 1950, des investissements publics conséquents ont permis d'augmenter la productivité de l'agriculture dans le Nordeste. Faisant suite à un effort d'industrialisation de la région, les investissements ont porté sur un large développement des infrastructures d'irrigation concentrées en grande partie dans la vallée du fleuve São Francisco. De 1970 à 1990, la taille des périmètres irrigués est passée de (116000 à 732000) ha, dont $32 \%$ situés dans cette vallée [3]

L'exploitation de ces zones a évolué au cours du temps. A l'origine, des cultures vivrières telles que le maïs, le haricot et parfois le riz inondé étaient dominantes. Dans un second temps, des productions telles que l'oignon et la tomate destinées à la vente en frais ou à la transformation ont été introduites [4]. Plus récemment, depuis les années 1980, l'arboriculture fruitière s'est fortement développée. Aujourd'hui, les périmètres irrigués de la vallée du São Francisco sont plantés principalement en manguiers, vignes, cocotiers, goyaviers et bananiers, productions récentes pour la région, destinées en partie à l'exportation.

Le pôle de production de fruits de Petrolina / Juazeiro, situé entre les États de Bahia et de Pernambuco dans la moyenne vallée du São Francisco, produit annuellement près de $926600 \mathrm{t}$ sur une surface de 50000 ha, dont 12000 ha occupés par des manguiers [5]. Il contribue à près de $70 \%$ des exportations de mangues destinées aux marchés européen et américain [3].

La région de Petrolina / Juazeiro revêt donc aujourd'hui une importance toute particulière pour la production de mangues. Dans cette région, la variété " Tommy Atkins " (figure 1) est préférée pour ses excellentes qualités agronomiques. Sa réponse à l'induction florale artificielle est bonne; la production est élevée et non alternante. Par ailleurs, la variété résiste bien au transport et aux manipulations après récolte. Néanmoins, ses qualités organoleptiques sont faibles et sa teneur en fibres est légèrement supérieure à celle d'autres variétés. Elle est sensible à un désordre physiologique : le jelly seed. Cette variété représente 83,6\% des surfaces plantées en manguiers dans les États de Bahia et de Pernambuco. Les autres variétés présentes sont "Haden ", "Espada " et "Keitt " avec, respectivement, 8,3\%, 1,7\% et 1,5\% des superficies plantées en manguiers ${ }^{1}$.

Paradoxalement, dans un contexte d'agriculture irriguée, les conditions climatiques du Sertão semi-aride apparaissent aujourd'hui comme un réel avantage pour certaines cultures fruitières. En effet, elles associent un ensoleillement important $\left(3000 \mathrm{~h} \cdot \mathrm{an}^{-1}\right)$ à une faible hygrométrie et à

\footnotetext{
${ }^{1}$ Cadastro fruticola, http://www.codevasf.gov.br
} 


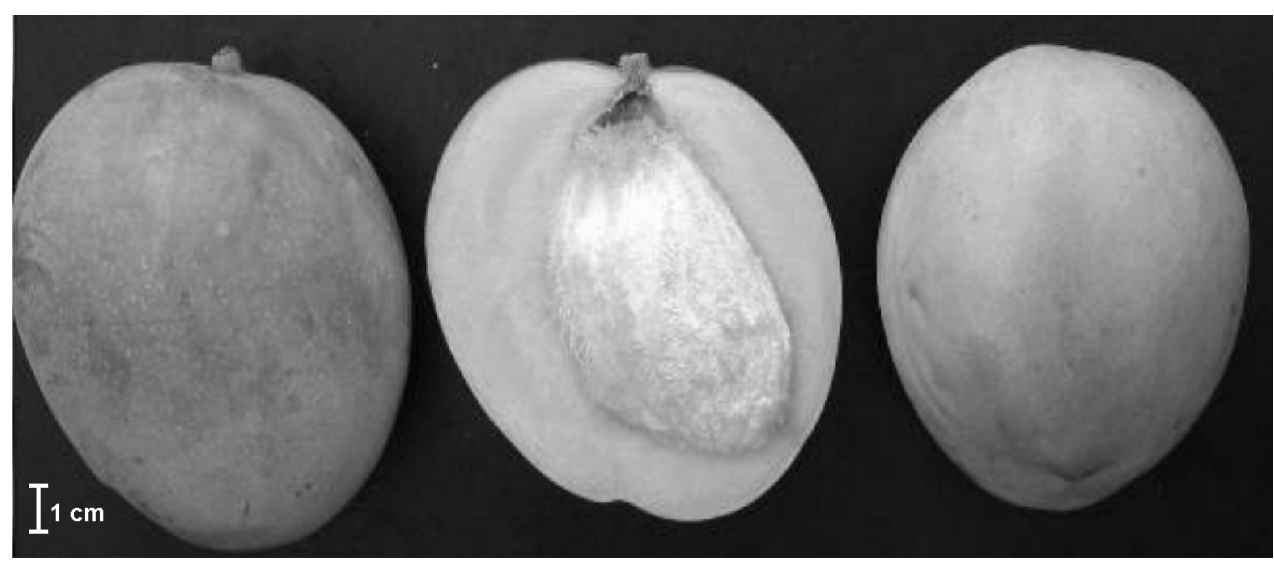

des températures moyennes de $26^{\circ} \mathrm{C}$ relativement constantes au cours de l'année [5]. Il en résulte une faible pression parasitaire et la possibilité de planifier, grâce à l'induction florale, une récolte de mangues à différentes époques de l'année [6]. Les producteurs de la vallée du São Francisco peuvent ainsi étaler leur production et approvisionner le marché international en ciblant la période où la concurrence des autres pays est minimale. La mangue du Nordeste brésilien a donc un avantage concurrentiel certain qui lui permet d'obtenir les meilleurs prix sur le marché américain.

La filière mangue a connu une période de développement euphorique qui s'est traduite dans un premier temps par une progression significative des plantations. En 2001, dans les seuls états de Bahia et de Pernambuco, $63 \%$ des vergers étaient jeunes et n'avaient pas encore atteint le stade de pleine production. La production régionale de mangues pourrait donc presque tripler dans les années à venir (figure 2). Ce phénomène associé à la montée en puissance de la production dans d'autres pays exportateurs pourrait fragiliser l'ensemble de la filière.

D'autre part, les exigences des pays importateurs sont de plus en plus strictes en raison de l'inquiétude croissante des consommateurs européens et américains vis-à-vis de l'innocuité des aliments. Actuellement, l'Union européenne uniformise sa législation concernant les limites maximales de résidus (LMR) pesticides autorisés sur les fruits et augmente ses exigences en

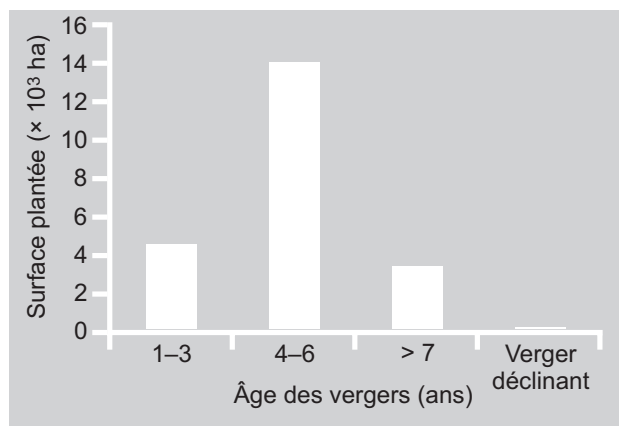

terme de traçabilité des produits. Depuis plusieurs années, les mangues destinées au marché nord-américain subissent un traitement hydrothermique rigoureux, imposé pour éviter l'entrée de la mouche des fruits (Anastrepha spp., Ceratitis capitata) aux Etats-Unis [7]. Plus récemment, les contrôleurs américains ont étendu leur préoccupation à l'organisation générale et à la propreté des stations d'emballage et les États-Unis sont de plus en plus demandeurs de certifications et de garanties sanitaires complémentaires.

\subsection{Objectifs de l'étude}

Face à ces perspectives, les producteurs et les agents de la recherche sont conscients de la nécessité de s'adapter aux futures contraintes du marché, de garantir la satisfaction d'acheteurs amenés à être d'autant plus exigeants que la concurrence sera rude. Bien des interrogations convergent vers les aspects "qualité du produit " pris au sens large.
Figure 1.

Mangue Tommy Atkins.

Figure 2.

Surface occupée par les vergers de manguiers dans les États de Bahia et de Pernambouc dans la région du Nordeste au Brésil (Cadastro fruticola, Codevasf 2000). 
Dans un contexte de collaboration scientifique entre la France et le Brésil et afin de participer à une évolution nécessaire de la région de production de fruits de Petrolina / Juazeiro, le Cirad-Flhor ${ }^{2}$ et l'Embrapa semiarido de Petrolina (CPATSA) souhaiteraient mettre en place, en partenariat, un programme de recherche et de développement qui s'inscrive au sein de la filière mangue du Vale do São Francisco. Cette opération devrait permettre d'accompagner les différents agents de la filière " manguier " dans leur adaptation aux profondes mutations en cours. Pour cette raison, le Cirad-Flhor a souhaité établir un diagnostic préalable afin de recueillir des informations précises sur les problèmes de qualité rencontrés par les producteurs du pôle Petrolina / Juazeiro et par les acteurs de la filière en aval, tant pour le marché interne que pour celui de l'exportation.

Dans les travaux qui vont être présentés, la qualité a été définie comme "une conformité aux exigences du client" [8]. Or, dans une filière, chaque maillon aval de la filière est le client de l'opérateur amont. Par conséquent, il était important de comprendre les exigences de chaque opérateur en terme de qualité pour savoir comment celle-ci s'élabore et fluctue, et comment elle est appréhendée par chacun.

L'étude entreprise a alors permis de caractériser les déterminants de la qualité dans la région d'étude. Elle a conduit à décrire tout d'abord l'organisation générale des circuits de distribution de la mangue produite dans la zone de Petrolina / Juazeiro, puis, dans un deuxième temps, elle a mis en évidence les coûts associés à la production et à la distribution pour les principaux débouchés relevés par le premier volet de la démarche. Enfin, nos travaux ont cherché à définir les caractéristiques d'une mangue de qualité telles qu'elles sont perçues dans la région d'étude pour chacun des débouchés identifiés et ils ont permis d'identifier les opérateurs qui imposent

${ }^{2}$ Cirad-Flhor : Département des productions fruitières et horticoles du Centre de coopération internationale en recherche agronomique pour le développement, Montpellier, France. leurs critères de qualité à la filière. Les stratégies sous-jacentes au choix de tel ou tel circuit de distribution par les opérateurs en amont de la filière ont alors été mieux cernées et nous avons pu évaluer comment la notion de qualité s'insérait dans ces stratégies.

\section{Matériel et méthodes}

L'étude a été réalisée durant l'été 2001, au travers d'enquêtes auprès de différents acteurs de la filière de production et de distribution de la mangue.

Onze producteurs de mangues ont été interrogés dont cinq ayant une petite exploitation de moins de 20 ha, deux disposant de parcelles de (20 à 100) ha et quatre exploitants de domaines de plus 100 ha. Par ailleurs, trois responsables de stations d'emballage, neuf grossistes des marchés de São Paulo et de Petrolina / Juazeiro, trois acheteurs de la grande distribution, six importateurs européens, six chercheurs de l'Embrapa, des transporteurs et de nombreux responsables d'institutions publiques ou non, liées à la culture fruitière, ont répondu à l'enquête.

Les enquêtes auprès des producteurs ont été réalisées sur les périmètres de Maniçoba, Nilo Coelho, Mandacaru, Bebedouro, Tourão, et Curaça du pôle Petrolina / Juazeiro situé dans la vallée sub-médiane du fleuve São Francisco et englobant les huit villages (municipes) attenants à ces deux villes. Ce pôle d'une surface de $24385 \mathrm{~km}^{2}$ comprend sept secteurs irriguées. En 1996, sur les périmètres irrigués du pôle Petrolina / Juazeiro, les petits producteurs [(20 à 100) ha] représentaient $30 \%$ de la surface totale plantée en manguiers [3].

Les enquêtes réalisées auprès de grossistes ont été menées sur deux marchés de gros différents, celui de Petrolina / Juazeiro et celui de São Paulo (CEAGESP ${ }^{3}$ ), le plus important marché de gros du Brésil. En

\footnotetext{
3 CEAGESP : Socióloga da Companbia de Entrepostose Amazéns Gerais de São
} Paulo. 


\begin{tabular}{|c|c|c|c|}
\hline $\begin{array}{l}\text { Données générales sur la ferme } \\
\text { et caractéristiques du verger de } \\
\text { manguiers }\end{array}$ & $\begin{array}{l}\text { Techniques de production } \\
\text { des mangues } \\
\text { et coûts associés }\end{array}$ & $\begin{array}{l}\text { Données liées à la } \\
\text { commercialisation } \\
\text { des mangues }\end{array}$ & $\begin{array}{l}\text { Gestion de la qualité des } \\
\text { mangues }\end{array}$ \\
\hline $\begin{array}{l}\text { Nom et date de création de la ferme } \\
\text { Nombre et type d'employés selon } \\
\text { les périodes de l'année } \\
\text { Superficie totale (ha) } \\
\text { Types de productions de la ferme } \\
\text { Superficies (ha) par type de } \\
\text { production } \\
\text { Inventaire des infrastructures et des } \\
\text { opérations mécanisées } \\
\text { Variétés de mangues cultivées } \\
\text { Densité et âge des arbres } \\
\text { Caractéristiques pédologiques du } \\
\text { verger } \\
\text { Date de récolte de la production de } \\
\text { mangues } \\
\text { Rendement sur les deux dernières } \\
\text { années }\end{array}$ & $\begin{array}{l}\text { Type d'irrigation et coût de revient } \\
\text { Technique d'induction florale } \\
\text { Type de fumure } \\
\text { Technique de lutte contre les } \\
\text { adventices } \\
\text { Type de protection phyto-sanitaire } \\
\text { Technique de cueillette } \\
\text { Opérations successives lors de la } \\
\text { cueillette } \\
\text { Critères de choix pour le point de } \\
\text { cueillette } \\
\text { Prise en compte de l'expérience } \\
\text { des cueilleurs } \\
\text { Traitement post-cueillette réalisé } \\
\text { sur la fazenda } \\
\text { Transport de la production }\end{array}$ & $\begin{array}{l}\text { Périodes de vente des } \\
\text { mangues } \\
\text { Destination des ventes } \\
\text { Volumes de vente pour } \\
\text { chacune de ces } \\
\text { destinations } \\
\text { Degré de } \\
\text { contractualisation des } \\
\text { relations du producteur } \\
\text { avec le client direct } \\
\text { Prix moyens pouvant } \\
\text { être obtenus sur les } \\
\text { différents marchés }\end{array}$ & $\begin{array}{l}\text { Définition de la qualité } \\
\text { Méthodologie de sélection } \\
\text { des fruits sur la fazenda } \\
\text { Critères de qualité supposés } \\
\text { selon les débouchés } \\
\text { Problèmes rencontrés } \\
\text { concernant la qualité } \\
\text { Opérations techniques favorisant } \\
\text { la qualité en fonction des } \\
\text { débouchés } \\
\text { Opérations effectivement } \\
\text { réalisées pour obtenir une } \\
\text { meilleure qualité } \\
\text { Type d'information désirée }\end{array}$ \\
\hline
\end{tabular}

1999, 68574 t de mangues y ont transité aussi bien en provenance de l'État de São Paulo que de la région du Nordeste [9].

Des questionnaires différents ont été élaborés pour les producteurs et les grossistes (tableaux I, II).

Par ailleurs, des observations ont été effectuées in situ au niveau des vergers, des stations d'emballage, des plates-formes de distribution et des marchés de gros. L'organisation générale, le chargement et le déchargement des fruits, la cohérence des méthodes de contrôle des lots ont été analysées.

Les enquêtes auprès des importateurs, des chercheurs et des responsables administratifs des institutions en rapport avec la culture fruitière ont été réalisées au moyen d'entretiens ouverts non directifs.

La décomposition des coûts de production, de transport et de distribution a été élaborée lors des enquêtes à partir de questions précises portant sur les coûts de chaque opération; les résultats obtenus ont été ensuite recoupés avec des données publiées [9].
La répartition des flux de mangues sur les différents marchés et la quantification en pourcentage ont été estimées grâce aux données transmises par diverses organisations : structures de production (VALEXPORT $^{4}$ et SIC-Vale ${ }^{5}$ ), grossistes des marchés de Juazeiro et de São Paulo, ainsi que des plates-formes de distribution, informations éditées par le ministère de l'Intégration nationale [10] et diverses autres données publiées [9, 11].

En raison de l'écrasante prédominance de la mangue Tommy Atkins par rapport aux autres variétés exploitées dans la zone d'étude, la plupart des données collectées n'ont porté que sur cette variété.

Toutes les données chiffrées ont été données en reals (monnaie brésilienne). Elles ont été calculées sur la base d'un

\footnotetext{
${ }^{4}$ VALEXPORT : Associação de Produtores e Exportadores de Hortigranjeiros e Derivados de Vale de São Francisco.

5 SIC-VALE : Sistema Integrados de Commercialização do Vale do São Francisco.
} 
Tableau II.

Informations recueillies par le biais de formulaires d'enquête auprès des grossistes assurant la commercialisation de la production de mangues récoltées dans la région du Nordeste (Brésil).

\begin{tabular}{|c|c|c|c|}
\hline $\begin{array}{l}\text { Données générales } \\
\text { sur l'entreprise }\end{array}$ & Relations amont-aval & $\begin{array}{l}\text { Opérations réalisées sur } \\
\text { le site de production } \\
\text { et coûts associés }\end{array}$ & Qualité et perspectives \\
\hline $\begin{array}{l}\text { Nom et date de création de } \\
\text { l'entreprise } \\
\text { Nombre et type } \\
\text { d'employés } \\
\text { Activités de l'entreprise } \\
\text { (vente, production, etc.) } \\
\text { Produits commercialisés } \\
\text { Volumes annuels } \\
\text { commercialisés } \\
\text { Types de débouchés } \\
\text { Volumes de mangues vendus } \\
\text { pour chaque débouché }\end{array}$ & $\begin{array}{l}\text { Fournisseurs et clients selon } \\
\text { les périodes de l'année } \\
\text { Modalités de paiement } \\
\text { (commission, prix fixe) } \\
\text { Degré de liberté dans } \\
\text { la négociation du prix } \\
\text { Degré de contractualisation } \\
\text { des relations avec les clients } \\
\text { Degré de connaissance } \\
\text { du client et du fournisseur } \\
\text { Fourchettes de prix de vente } \\
\text { selon les périodes de l'année }\end{array}$ & $\begin{array}{l}\text { Conditions et coût de } \\
\text { transport jusqu'au } \\
\text { grossiste } \\
\text { Opérations de } \\
\text { chargement et } \\
\text { déchargement } \\
\text { Temps et conditions } \\
\text { de stockage sur le marché } \\
\text { de gros } \\
\text { Conditions de transport } \\
\text { jusqu'au client }\end{array}$ & $\begin{array}{l}\text { Critères pour juger de la qualité } \\
\text { des mangues } \\
\text { Problèmes liés à la qualité des mangues } \\
\text { Degré de tolérance pour chaque critère } \\
\text { Fréquence d'occurrence des problèmes } \\
\text { Conséquences des problèmes } \\
\text { Relation entre qualité des mangues } \\
\text { et prix de vente } \\
\text { Pénalité liée au manque de qualité } \\
\text { Liste des problèmes à résoudre } \\
\text { Enjeux d'une mangue de qualité } \\
\text { Besoins d'une meilleure qualité } \\
\text { Informations / formations nécessaires } \\
\text { Intérêt des normes de classification }\end{array}$ \\
\hline
\end{tabular}

cours moyen de 0,46 \$US ou 0,5 euros par réal sur la période de l'été 2001.

\section{Résultats et discussion}

\subsection{Circuits de distribution de la mangue de la zone de Petrolina / Juazeiro}

De premiers résultats ont permis de mettre en évidence l'organisation générale de la distribution des mangues produites dans la vallée du São Francisco. Trois débouchés différents s'offrant aux producteurs de cette vallée ont été identifiés : commercialisation sur le marché brésilien, exportation vers l'Amérique et exportation vers L'Europe. Les quantités relatives (\%) de mangues dirigées vers l'un ou l'autre de ces circuits de commercialisation ont été identifiées (figure 3).

Cette analyse a révélé que la majeure partie des mangues produites sur le pôle Petrolina / Juazeiro (55\%) transitait par les marchés de gros locaux ou par ceux d'autres régions du Brésil. Le producteur peut faire le choix de vendre directement aux grossistes ou de passer par un négociant intermédiaire. Environ $35 \%$ des mangues produites sont destinées à l'exportation. Nous avons pu observer que les circuits de commercialisation sur le marché interne, passant par de multiples intermédiaires, pouvaient parfois être très longs. Le grand nombre de manipulations alors nécessaire, augmentant d'autant les risques de dommages physiques, peut être très préjudiciable à la qualité des fruits commercialisés au stade du détail.

Dans la mesure du possible, les producteurs de mangues du pôle Petrolina / Juazeiro tentent d'alimenter les trois circuits évoqués précédemment, avec, cependant, une préférence pour ceux d'exportation. Pour cela, ils adoptent diverses stratégies qui sont fonction de la taille de leur exploitation, de leur pouvoir de négociation et de leur capacité d'investissement. Le marché interne est le moins risqué de tous puisque les investissements à réaliser sont peu importants, les mangues étant alors souvent directement pesées et emballées au verger, juste après leur cueillette. Mais ce marché interne est aussi celui qui présente les moindres profits. Plus précisément, c'est un marché qui est rémunérateur lorsque les mangues sont produites en période de pénurie sur le marché interne (de mars à août principalement) et que les prix sont au plus haut (figure 4). 


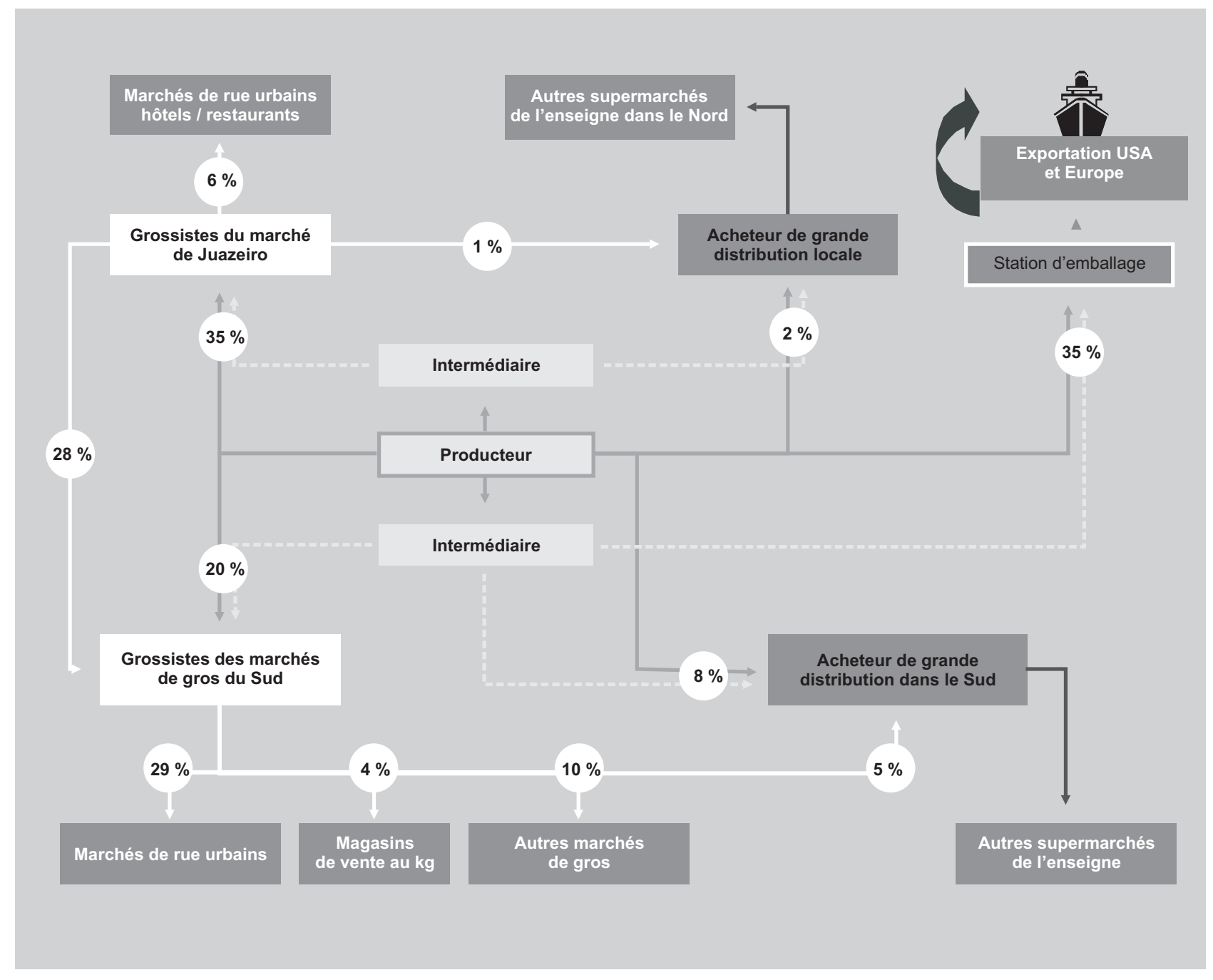

A contrario, le marché américain présente des risques beaucoup plus importants, tant en terme d'investissements à réaliser pour pouvoir exporter vers ce pays qu'en raison des variations potentielles du prix, mais il peut être extrêmement rémunérateur. Les installations nécessaires pour le traitement thermique contre la mouche des fruits sont très onéreuses. La station de conditionnement doit être accréditée tous les ans et prendre en charge le coût d'un agent de l'USDA (United States Department of Agriculture) qui vérifie la bonne exécution des traitements durant toute la saison d'exportation. Tout cela augmente considérablement les prix de revient d'un kilo de mangues destinées à l'exportation vers les États-Unis.
Les bénéfices pouvant être espérés sur le marché européen sont légèrement moindres que ceux du marché américain ; ils restent néanmoins plus intéressants que ceux du marché interne. En effet, rarement

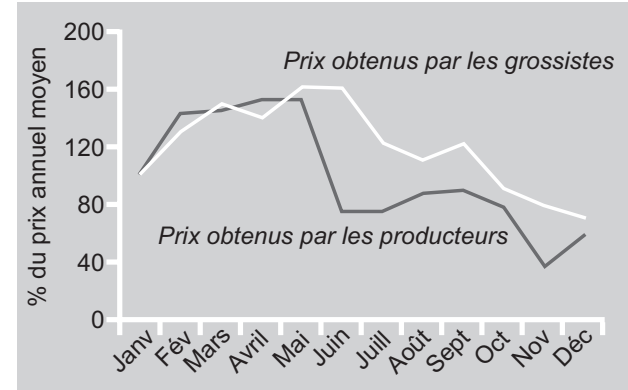

Figure 3.

Circuits de commercialisation des mangues produites dans la zone de Petrolina / Juazeiro, région du Nordeste, Brésil.

\section{Figure 4.}

Indices des prix mensuels obtenus par les producteurs et les grossistes du marché de gros de São Paulo (CEAGESP) par rapport au prix moyen pratiqué dans l'année (Nordeste, Brésil). 


\begin{tabular}{|c|c|c|c|}
\hline Poste considéré & $\begin{array}{c}\text { Marché interne } \\
\left(\text { real } \cdot \mathrm{kg}^{-1}\right)\end{array}$ & $\begin{array}{c}\text { Europe } \\
\left(\text { real } \cdot \mathrm{kg}^{-1}\right)\end{array}$ & $\begin{array}{l}\text { États-Unis } \\
\left(\text { real } \cdot \mathrm{kg}^{-1}\right)\end{array}$ \\
\hline Production & 0,2 & 0,3 & 0,3 \\
\hline $\begin{array}{l}\text { Cueillette, traitements et } \\
\text { emballage }\end{array}$ & 0,15 & 0,32 & 0,5 \\
\hline $\begin{array}{l}\text { Transport à la station de } \\
\text { conditionnement }\end{array}$ & 0,01 & 0,01 & 0,01 \\
\hline Achat des cartons & 0,1 & 0,1 & 0,1 \\
\hline Transport routier & 0,07 & - & - \\
\hline Commission station d'emballage & 0,06 & - & - \\
\hline Pertes (10 \%) & 0,06 & - & - \\
\hline Déchargement & 0,01 & - & - \\
\hline $\begin{array}{l}\text { Commission des grossistes } \\
(12 \%)\end{array}$ & 0,09 & - & - \\
\hline Transport jusqu'au port & - & 0,1 & 0,1 \\
\hline Taxe de commercialisation & - & 0,05 & 0,05 \\
\hline Chambre froide au port & - & 0,05 & 0,05 \\
\hline Embarquement & - & 0,03 & 0,03 \\
\hline Fret maritime & - & 0,38 & 0,5 \\
\hline Manutention & - & 0,12 & 0,12 \\
\hline Taxes d'importation & - & 0,113 & 0,11 \\
\hline Déchargement & - & 0,06 & 0,06 \\
\hline $\begin{array}{l}\text { Commission de l'importateur } \\
(12 \%)\end{array}$ & - & 0,1 & 0,13 \\
\hline Total & 0,75 & 1,75 & 2,0 \\
\hline $\begin{array}{l}\text { Echelle de prix sur marchés de } \\
\text { destination }\end{array}$ & $0,71-2$ & $2-3$ & $2-5$ \\
\hline Marge potentielle & $0-1,25$ & $0,25-1,25$ & $0-3$ \\
\hline
\end{tabular}

les meilleurs prix obtenus sur le marché brésilien n'atteignent ceux, assez stables, observés sur le marché européen.

La décomposition des coûts de production mise en relation avec l'estimation des prix pouvant être obtenus permet de mettre en évidence les caractéristiques des différents marchés en terme de rémunération potentielle et de risque (tableau III).

\subsection{Déterminants de la qualité}

Pour mettre en évidence les déterminants de la qualité des mangues produites dans la région de Petrolina / Juazeiro, nous avons distingué deux types de critères, ceux relatifs à l'aspect extérieur des fruits, et ceux caractérisant le stade de maturité en relation avec la complexité de la phase de mise en marché.

Les critères de qualité considérés par les grands producteurs comme les stations de conditionnement et les exportateurs de la région de Petrolina / Juazeiro ont été identifiés (tableau IV). Les normes éditées par l'OCDE $^{6}$ [12] sont représentatives des exigences générales en terme de qualité des

\footnotetext{
${ }^{6}$ Organisation de coopération et de développement économiques, Paris, France.
} 
Tableau IV.

Déterminants d'une mangue (variété Tommy Atkins) de qualité pour les différents marchés, tels qu'ils sont définis par les producteurs et stations d'emballages de la région de Petrolina / Juazeiro (Nordeste, Brésil).

\begin{tabular}{|c|c|c|c|c|}
\hline \multicolumn{2}{|c|}{ Déterminants de la qualité } & Marché interne & Europe & États-Unis \\
\hline \multirow[t]{7}{*}{$\begin{array}{l}\text { Aspect } \\
\text { extérieur }\end{array}$} & $\begin{array}{l}\text { Maturité lors de la récolte } \\
\text { Calibre }\end{array}$ & $\begin{array}{c}\text { Stade } 3 \\
\text { Tous calibres selon } \\
\text { les acheteurs }\end{array}$ & $\begin{array}{l}\text { Stade } 2 \text { à } 2,5 \\
5 \text { à } 14^{2}\end{array}$ & $\begin{array}{l}\text { Stade } 2,5 \\
7 \text { à } 12^{2}\end{array}$ \\
\hline & Degré Brix & 7 à 8 & 6,5 à 8,5 & 7 à 7,5 \\
\hline & Fermeté & Peu ferme & Très ferme & Moyenne \\
\hline & Coloration & (10 à 35) \% & $35 \%$ & $50 \%$ \\
\hline & Trace de latex & Tolérance & Absence & Absence \\
\hline & Brûlures (froid ou soleil) & $\begin{array}{c}\text { Tolérance si } \\
\text { incidence faible }\end{array}$ & Absence totale & Absence totale \\
\hline & Coups & Absence & Absence totale & Absence totale \\
\hline \multirow[t]{3}{*}{$\begin{array}{l}\text { Aspect } \\
\text { parasitaire }\end{array}$} & Anthracnose & $\begin{array}{c}\text { Tolérance si } \\
\text { incidence }<5 \%\end{array}$ & $\begin{array}{l}\text { Tolérance faible, } \\
\text { variable selon } \\
\text { acheteurs }\end{array}$ & $\begin{array}{l}\text { Tolérance faible, } \\
\text { variable selon } \\
\text { acheteurs }\end{array}$ \\
\hline & $\begin{array}{l}\text { Marques d'attaque } \\
\text { parasitaire }\end{array}$ & Absence & Absence & Absence totale \\
\hline & $\begin{array}{l}\text { Larves de mouche } \\
\text { des fruits }\end{array}$ & Absence totale & Absence totale & Absence totale \\
\hline \multirow[t]{2}{*}{ Aspect interne } & Collapse interne & Absence & Absence & Absence \\
\hline & $\begin{array}{l}\text { Pourriture du pédoncule, } \\
\text { nez mou, etc. }\end{array}$ & Absence & Absence & Absence \\
\hline \multirow[t]{3}{*}{$\begin{array}{l}\text { Aspect } \\
\text { sanitaire }\end{array}$} & $\begin{array}{l}\text { Conformité des résidus de } \\
\text { pesticides aux normes en } \\
\text { vigueur }\left(\mathrm{LMR}^{3}\right)\end{array}$ & Peu de contrôle & $\begin{array}{l}\text { Exigence forte, } \\
\text { contrôles }\end{array}$ & Exigence forte \\
\hline & Traçabilité des lots & Peu d'exigences & Exigence forte & Exigence forte \\
\hline & $\begin{array}{c}\text { Conformité des } \\
\text { installations des stations } \\
\text { d'emballage aux normes }\end{array}$ & Pas d'exigences & Peu d'exigences & Exigence forte \\
\hline \multirow{3}{*}{$\begin{array}{l}\text { Aspect de } \\
\text { présentation }\end{array}$} & Uniformité des cartons & Tolérance de $20 \%$ & Exigence forte & Exigence forte \\
\hline & & $\begin{array}{l}\text { immédiatement } \\
\text { supérieur par carton }\end{array}$ & & \\
\hline & Aspect des palettes & - & Exigence forte & Exigence forte \\
\hline
\end{tabular}

${ }^{1}$ Définie selon l'échelle en vigueur dans la région d'étude allant de 0 (fruits totalement verts) à 5 (fruits très mûrs). Le stade 2 correspond à un commencement de jaunissement de la pulpe.

${ }^{2}$ Calibre pour une caisse de $4 \mathrm{~kg}$ (norme exprimant le nombre de fruits par caisse).

${ }^{3}$ LMR : limite maximale de résidus.

${ }^{4}$ Normes imposées par le pays importateurs (exemple : normes USDA pour le traitement

hydrothermiques des fruits, ou audits imposés par les standards d'innocuité des aliments).

fruits pour le commerce international. Néanmoins, selon les destinations, les exigences des acheteurs diffèrent quelque peu. Les importateurs européens attachent une importance toute particulière à la fer- meté des fruits et au respect des normes sur les limites maximales de résidus. Les acheteurs américains sont plus exigeants quant à la coloration des fruits et ne tolèrent pas les calibres trop gros ou trop petits. Leurs 
exigences sont bien entendu très strictes en ce qui concerne la conformité des installations des stations de conditionnement, tout particulièrement en ce qui concerne le traitement hydrothermique des fruits.

La gestion du mûrissement des fruits, elle-même en grande partie dépendante de la logistique d'acheminement tout au long de la filière, influe fortement sur la qualité finale. Les attentes du consommateur ne sont prises en compte que partiellement et, souvent, lors des arbitrages, elles ne sont pas prioritaires.

Le choix de la variété Tommy Atkins est une bonne illustration des critères de choix retenus. Son exploitation préférentielle a été motivée par des raisons agronomiques et par la tolérance de ce fruit aux conditions de transport ; il n'a pas alors été tenu compte de ses qualités gustatives. Or, le consommateur européen est particulièrement sensible à la qualité organoleptique qui est l'un des critères qui permet de fidéliser son choix [13] et les importateurs européens sont sensibles à la fermeté des mangues, car cela permet de réduire les risques d'une mauvaise conservation lors du transport et de la mise en marché. Cette dernière exigence se traduit trop souvent par une cueillette à un stade trop précoce défavorable à une maturation optimale garante d'une bonne qualité gustative.

En effet, la mangue est un fruit climactérique qui poursuit son processus de maturation après récolte, à la condition que le fruit soit cueilli après un stade précis de son évolution sur l'arbre. Récolté trop tôt, le fruit ne mûrira pas ; récolté trop tard, son évolution rapide sera incompatible avec les contraintes de conservation. La définition du point de cueillette des mangues est donc un élément essentiel pour la gestion de la qualité. Pour le moment, il n'existe encore aucune technique fiable permettant de s'assurer que les mangues sont au stade optimal de maturité pour être récoltées. Le nombre de jours de développement du fruit est probablement l'un des critères les plus pertinents à prendre en compte (environ $105 \mathrm{j}$ pour la mangue Tommy Atkins). L'hétérogénéité de l'âge des fruits et de leur environnement au sein de l'arbre et du verger et l'impossibilité de contrôler chaque fruit sont autant de frein à la mise au point d'une méthode de prévision de récolte [14].

Durant la saison d'exportation, le marché est tendu ce qui se traduit par une forte réactivité des cours d'un jour à l'autre. Dans ces conditions, les producteurs sont souvent tentés de récolter leurs fruits pour satisfaire la demande en négligeant la qualité. Cette attitude se traduit par des critiques de plus en plus nombreuses de la part des importateurs et distributeurs et par des litiges commerciaux fort coûteux pour les stations de conditionnement. Pour ces raisons, la campagne 2001-2002 s'est révélée commercialement catastrophique.

\section{Conclusion}

\subsection{Obtention de mangues de meilleure qualité}

Pour améliorer la qualité des mangues, il serait nécessaire de prendre en compte les résultats de recherche obtenus à partir de l'étude des différentes étapes de l'élaboration puis de la conservation de cette qualité. La définition du point de coupe sur l'arbre, la constitution de lots de fruits de maturité homogène au moment de la récolte ou en station de conditionnement, la gestion des échanges gazeux après la récolte sont autant de points clés pouvant influencer la qualité des fruits commercialisés.

Pour mieux connaître le stade de la maturité du fruit au moment de la récolte, plusieurs démarches complémentaires seraient nécessaires :

- la formation des cueilleurs à la prise en compte de déterminants visuels de la maturité ;

- le développement de techniques fiables, non destructives, mesurant des paramètres physiologiques du fruit, utiles pour identifier le point de coupe. Dans ce domaine, plusieurs approches basées sur des procédés physiques comme l'acoustique impulsionnelle ou la spectrométrie proche infrarouge pourraient s'avérer intéressantes [15]. Cela permettrait de cueillir les fruits à un 
stade de maturation optimale, défini à partir de l'analyse d'un échantillonnage fiable, associé à une caractérisation non destructive de la maturité. À terme, les stations de conditionnement seraient alors aptes à constituer des lots de fruits dont le degré de maturité serait plus homogène. Lors de la conservation de la production, du transport et de la mise en marché, la gestion de ces lots serait plus aisée.

Pour améliorer le contrôle de la maturation du fruit après la coupe tout en cherchant à prolonger la phase de conservation sans préjudice pour la qualité, certaines précautions pourraient être prises :

- respect plus scrupuleux de la chaîne du froid pendant toute la période allant de la récolte à la mise en marché ;

- utilisation d'enrobages ou de films à perméabilité sélective créant une atmosphère modifiée au voisinage immédiat du fruit. Ces techniques ouvrent des perspectives prometteuses car le ralentissement alors constaté de l'évolution de la maturation permet de récolter des fruits plus matures dont le potentiel aromatique et gustatif est amélioré sans pour autant qu'il y ait de risque majeur de surmaturité au stade du commerce de détail [16].

\subsection{Besoin d'une rémunération de la qualité}

La situation récente de déséquilibre de l'offre et de la demande se traduit par de fortes variations de cours aussi bien sur le marché interne que sur les marchés d'exportation. Cela est particulièrement vrai pendant la période allant de mars à août. Les opérateurs de la région du Nordeste ont une approche commerciale opportuniste qui les conduit, dans ce cas, à négliger la qualité des produits. En effet, durant cette période, le marché interne est un marché plus rémunérateur qu'il n’y paraît et moins exigeant en terme de qualité que le marché d'exportation qui est alors plus contraignant et plus risqué car, outre des investissements plus importants, les pertes liées aux écarts de triage sont conséquentes. Il faut également prendre en compte les refus ou les baisses de prix pra- tiquées à destination en raison de la mauvaise qualité de certains lots de mangues. Ces difficultés n'incitent pas certains producteurs à s'impliquer dans une démarche cohérente de qualité structurée au sein de la filière.

Les résultats de la campagne 2000-2001 ont montré toutes les limites de cette approche opportuniste. À la fin de cette période, les stations de conditionnement se sont retrouvées dans une situation financière délicate en raison de nombreux litiges portant sur la qualité. Dans les prochaines années, la jeunesse actuelle des vergers se traduira nécessairement par une forte augmentation de la production et donc par la nécessité de trouver de nouveaux débouchés. Or, la concurrence des autres pays producteurs ne faiblit pas. Dans ce contexte, la démarche "qualité " doit être perçue comme un élément central sur lequel se bâtira une relation de confiance durable entre les producteurs et les consommateurs en impliquant l'ensemble des intermédiaires. Depuis 1999, un effort important a été entrepris par les professionnels et la recherche agronomique (Embrapa semi-arido de Petrolina), suite à l'impulsion donnée par les autorités gouvernementales. Un vaste programme de production fruitière intégrée est opérationnel. Les initiatives de production de mangues suivant les principes de l'agriculture biologique ont dépassé le stade expérimental et se développent sur des surfaces de plusieurs dizaines d'hectares chez les plus grands producteurs. Ces pratiques concernent essentiellement un aspect de la qualité : l'innocuité. Cependant, beaucoup reste à faire en matière de qualité organoleptique.

\subsection{Complexité de l'organisation}

Notre étude a permis de mettre en évidence la complexité de l'organisation de la filière "mangue " dans le Nordeste brésilien. Les circuits de commercialisation sont longs et ramifiés, ce qui induit un morcellement des activités et des responsabilités. Beaucoup de producteurs et d'exportateurs ne perçoivent pas l'intérêt d'une coordination et se font concurrence. En raison de l'éloignement entre les marchés de 
consommation et les centres de production, il serait nécessaire d'établir des rapports sains entre les opérateurs qui pourraient s'appuyer sur des critères fiables communs à chaque parti pour apprécier la qualité de la marchandise proposée et sécuriser les conditions d'acheminement.

Par ailleurs, il convient de noter que le système de vente à la commission est très communément pratiqué. Dans ce cas, les mangues sont expédiées sur le marché d'importation à un commissionnaire vendeur qui va vendre au meilleur prix mais sans prise de risque, c'est-à-dire parfois en dessous du prix de revient. Les producteurs brésiliens qui adoptent ce système de vente perdent toute possibilité de maîtrise du marché, élément surprenant pour une région où la production exportée pourrait être aisément contrôlée.

Une meilleure coordination des opérateurs, en particulier de ceux qui interviennent dans le marché d'exportation, permettrait de mieux identifier les attentes des consommateurs et de gérer des relations commerciales sur le long terme. Dans d'autres pays et pour d'autres produits, il existe ainsi plusieurs exemples d'organisations concentrées d'exportateurs de fruits frais exotiques : le New Zealand Kiwi Marketing Board (NZKMB) pour le kiwi en Nouvelle-Zélande avec la marque 'Zespri', CAPESPAN $^{7}$ International en Afrique du Sud avec respectivement la marque 'Cape' pour les fruits tempérés et 'Outspan' pour les agrumes, le Citrus Marketing Board of Israël (CMBI) avec la marque 'Jaffa', etc. À l'origine, toutes ces structures ont eu comme vocation commune de structurer l'offre et de faire contrepoids aux importateurs. Elles se sont organisées autour du goulot d'étranglement que constitue l'expédition des fruits (conditionnement, transport, mise en marché) avec une double préoccupation :

- maintien des cours à un niveau satisfaisant en évitant de se présenter en ordre dispersé devant les centrales d'achats et les importateurs ;

\footnotetext{
${ }^{7}$ CAPESPAN : nom contracté suite à un joint-venture entre Oustpan International et Unifruco Ltd.
}

- respect attentif de normes pour la mise en marché de produit de qualité et la satisfaction des clients (intermédiaires de la distribution et consommateurs).

Une telle structuration s'est révélée particulièrement performante pour développer sous une même marque une politique commerciale suivie, réguler l'offre en limitant les mises en marché, faire remonter vers l'amont, au niveau de la structure de production, les attentes des consommateurs et des distributeurs et organiser vers l'aval des campagnes de promotion et d'informations sur un produit labellisé [17, 18].

\section{Remerciements}

Nous souhaitons remercier l'Embrapa semiarido de Petrolina (CPATSA), le groupe Global Fruit, ainsi que le Centro de Qualidade em Horticultura (CQH) du marché de gros de São Paulo (CEAGESP) pour leur contribution à l'organisation de cette étude.

\section{Références}

[1] Pratini de Moraes M.V., Mapeamento da fruticultura brasileira, Ministerio da agricultura e do abastecimento, Brasilia, Brazil, 2000, $234 \mathrm{p}$.

[2] Braz J., La filière mangue au Brésil, FruiTrop 86 (2001) 2-10.

[3] Anonymous, Os casos das regiões de Petrolina-Juazeiro e Norte de Minas Gerais, Escrito Tecnico de estudos économicos do Nordeste, Banco do Nordeste, Fruticultura irrigada, Fortaleza, Brazil, 2000, 83 p.

[4] Marinozzi G., Stratégies collectives et dispositifs de commercialisation : l'essor de la fruticulture irriguée à Juazeiro Petrolina (Nordeste du Brésil), Cirad-Tera et INP Toulouse, thèse, Toulouse, France, 2000, 294 p.

[5] Araujo J.L.P, Correia R.C., Comportamento de preços da manga na região do submedio São Francisco, in: Comunicado Tecnico da Embrapa semi-Arido dez 2000, $\mathrm{n}^{\circ} 94$ Embrapa semi-Arido, Brazil, 2000, pp. 1-5.

[6] Silva de Albuquerque J.A., Do Carmo Mouco M.A., Manga: Indução floral, Circular tecnica da Embrapa Semi-Arido, set. 2000, $n^{\circ} 47$, 
Embrapa semi-Arido, Petrolina, Brazil, 2000, $34 \mathrm{p}$.

[7] Mendonca M.C., Nascimento A.S., Caldas R.C., Pereira Filho C.A., Effect of hydrothermal treatment of mango fruits on larval mortality of Ceratitis capitata (wied.) (Diptera: Tephritidae), An. Soc. Entomol. Bras. 29 (1) (2000) 139-145.

[8] Gomez P.Y., Qualité et théorie des conventions, Paris, Economica, 1994, 270 p.

[9] Anonymous, FNP Consultoria e comercio, Agrianual 2001, São Paulo, Brazil, www.fnp.com.br

[10] Anonymous, Frutifatos, informação para a fruticulture irrigada, uma perspectiva de mercado para a agricultura irrigada, Ministerio da integracao national, Brasilia, Brazil, Vol. 1 (1), sept. 1999, 23 p.

[11] Mandetta de Souza R.A., De Oliveira Pithan e Silva R., Mandelli C.S., Pagotto Tasco A.M.P., Commercialização horticola: análise de alguns setores do mercado varejista de São Paulo, Inf. Econ. SP 28 (10) (1998) 7-23.

[12] Anonyme, Normalisation internationale des fruits et légumes : mangues, Organisation de coopération et de développement économique (OCDE), Paris, France, 1993, 64 p.
[13] Armstrong R., European mango sales continue to soar, Eurofruit (mars) (2001) 64-74.

[14] Lederman I.E., Bezerra J.E.F., Carvalho de P.S., Alves M.A., Santos dos V.F., Determinação do ponto de colheita da Manga cv. Tommy Atkins para a região semi arida do Pernambuco, Rev. Bras. Frutic. 20 (2) (1998) 145-151.

[15] Valente M., Banzouzi J.T., Piétri E., Dornier E., Evaluation non destructive de la fermeté de la mangue par la technique acoustique impulsionnelle, Fruits 55 (5) (2000) 333-345.

[16] Ducamp-Collin N.N., Importance de la conservation sous atmosphère modifiée des fruits tropicaux: application à la mangue, FruiTrop 85 (2001) 19-20.

[17] Codron J.-M., Les stratégies d'approvisionnement de la grande distribution en produits frais. Cas des fruits de contre-saison, Inra, série Études et Recherches, $n^{\circ} 105$, Paris, France, 1996.

[18] Weinberg Y., Marketing of fresh citrus fruit, the market and citrus marketing board of Israel in the next decade, in: Goren R., Mendel K., Proc. 6th Int. Citrus Congr., Vol. 4, Balaban Publ. and Margraf Publ., Israel, 1988, pp. 1567-1570. 


\section{Determinantes de la calidad para el mango producido en el noreste brasileño.}

Resumen - Introducción. Desde el establecimiento, en los años 60, de extensas zonas de regadío en la región de Petrolina/Juazeiro (Nordeste, Brasil), ésta se convirtió en uno de los grandes polos brasileños de producción frutal y, en particular, de mangos destinados a la exportación. Sin embargo, la situación privilegiada de los productores de esta zona está amenazada por la perspectiva de una competencia muy fuerte en los mercados internos y de exportación. Los compradores podrían volverse más exigentes con respecto a la calidad de la fruta. Nuestro trabajo estudió la organización de la producción y la distribución de mangos para comprender cómo la calidad podía influir en las estrategias de los operadores de esta zona. Material y métodos. Una encuesta realizada durante 4 meses en el polo de Petrolina/ Juazeiro y en el mayor mercado mayorista brasileño en São Paulo permitió interrogar a 50 operadores del sector: productores, responsables de centros de embalajes, mayoristas, exportadores, importadores, científicos y representantes de instituciones públicas. Resultados y discusión. La distribución de los flujos de mangos producidos en Petrolina/Juazeiro indica que un alto porcentaje (65\%) de la producción se vende en el mercado interno y que el 55\% pasa por los mercados mayoristas. Los circuitos de intercambios sucesivos, que perjudican la calidad de la fruta, pueden ser muy largos. La descomposición de los costos de producción y comercialización muestra que, en algunos períodos del año, el mercado interno puede ser tan rentable como el mercado de exportación a Europa. El mercado americano es el que implica mayores riesgos debido a las importantes inversiones a realizar, pero potencialmente también es el más lucrativo de agosto a septiembre. La calidad de los mangos producidos en Petrolina/ Juazeiro se basa en su aspecto y, sobre todo, el control de la madurez. Conclusión. Actualmente los precios están más determinados por las cantidades disponibles en los mercados que por la calidad real de los mangos. Debido a la competencia futura, esta situación no debería durar y la calidad será esencial para mantener la competitividad en el mercado. Una mejor coordinación de los actores del sector así como el desarrollo de técnicas o formaciones para determinar mejor el momento de cosecha óptimo y controlar mejor el desarrollo de los frutos después del corte permitirían solucionar los problemas de calidad más graves.

Brasil / Mangifera indica (frutas) / producción / corrientes de mercadeo / calidad / información situación del mercado

To access this journal online: www.edpsciences.org 